\title{
Instabilities in the Ionization Zones Around the First Stars*
}

\author{
E. O. Vasiliev ${ }^{1,4 \dagger}$, E. I. Vorobyov ${ }^{1,2}$, A. O. Razoumov ${ }^{3}$, Yu. A. Shchekinov ${ }^{4,5}$ \\ ${ }^{1}$ Institute of Physics, Southern Federal University, Rostov-on-Don, Russia \\ ${ }^{2}$ Institute of Astronomy, University of Vienna, Austria \\ ${ }^{3}$ SHARCNET/UOIT Consortium, Oshawa, Canada \\ ${ }^{4}$ Physics Department, Southern Federal University, Rostov-on-Don, Russia \\ ${ }^{5}$ Special Astrophysical Observatory, Russian Academy of Sciences, \\ Nizhnyi Arkhyz, Karachay-Cherkessia Republic, Russia
}

\begin{abstract}
We consider the evolution of the ionization zone around Population III stars with $M_{*} \sim$ $25-200 M_{\odot}$ in protogalaxies with $M \sim 10^{7} M_{\odot}$ at redshifts $z=12$, assuming that the dark matter profile is a modified isothermal sphere. We study the conditions for the growth of instabilities in the ionization zones. The Rayleigh-Taylor and thermal instabilities develop efficiently in the ionization zones around $25-40 M_{\odot}$ stars, while this efficiency is lower for stars with $\sim 120 M_{\odot}$. For more massive stars $\left(\sim 200 M_{\odot}\right)$, the flux of ionizing photons is strong enough to considerably reduce the gas density in the ionization zone, and the typical lifetimes of stars $(\sim 2 \mathrm{Myr})$ are insufficient for the growth of instabilities. The gas in a protogalaxy with $M \sim 10^{7} M_{\odot}$ with a $200 M_{\odot}$ central star is completely ionized by the end of the star's lifetime; in the case of a $120 M_{\odot}$ central star, only one-third of the total mass of gas is ionized. Thus, ionizing photons from stars with $M_{*} \lesssim 120 M_{\odot}$ cannot leave protogalaxies with $M \gtrsim 10^{7} M_{\odot}$. If the masses of the central stars are 25 and $40 M_{\odot}$, the gas in protogalaxies of this mass remains essentially neutral. We discuss the consequences of the evolution of the ionization zones for the propagation of the envelope after the supernova explosions of the stars and the efficiency of enrichment of the intergalactic medium in heavy elements.
\end{abstract}

\section{Introduction}

Various instabilities can arise in the ionization zones around massive stars, related to the thermodynamics of the gas behind the ionization front and/or shock, as well as the break-out of the ionization front [1-3]. These instabilities lead to substantial fluctuations of the density and temperature and increase the velocity dispersion of the gas in the ambient interstellar medium (ISM). Hence, subsequent supernovae will occur in the medium with random flows, probably facilitating a rapid break-up of the supernovae envelopes and increasing the efficiency of mixing of heavy elements in the ISM [4]. These effects are not only important for the ISM [5], but are especially interesting for the problem of the primordial enrichment of the first protogalaxies by Population III stars.

The evolution of ionization zones around the first stars has been studied in some detail [6-9]. The main focus of these papers is the global dynamics of the propagation of the ionization front and the influence of the ionizing radiation of the first stars on the efficiency of photo-evaporation

\footnotetext{
${ }^{*}$ This paper is published in Astronomy Reports, 2012, Vol. 56, No. 7, pp. 564-571.

†eugstar@mail.ru
} 
of gas in the first protogalaxies, or in other words, on their destruction [10]. Another problem was the calculation of the number of ionizing photons leaving the protogalaxy, or the escape fraction [11, 12], which is important for studies of the possibility of observing the ionization zones [13] and the dynamics of the ionization of the intergalactic medium [14], and, hence, reionization of the Universe. It is evident that more accurate studies of the evolution of the propagation of the ionization fronts require investigation of the dynamics on small scales and the possible growth of instabilities in ionization zones around the first stars. The first attempt to investigate this problem using three-dimensional modeling was carried out in [15], which assumed that the initial density profile in the cloud followed a power-law, $\sim r^{-2}$ (steeper distributions were also studied, with the aim of investigating the growth of the Rayleigh-Taylor instability); to some extent, this assumption corresponds to the conditions in the first protogalaxies. However, note that the gas profile also depends on the dark-matter distribution in the protogalaxy, and more realistic studies of the efficiency of the growth of instabilities in the ionization zones taking into account the dynamics of gas cooling in the dark-matter potential are necessary.

Here, we study the growth of instabilities in ionization zones around the first massive stars in spheroidal (non-rotating) dwarf protogalaxies with the dark-matter density profile corresponding to a modified isothermal sphere. We used a cosmological model with a $\Lambda$-term and cold dark matter $\left(\Lambda \mathrm{CDM}\right.$ model) with $\left(\Omega_{0}, \Omega_{\Lambda}, \Omega_{m}, \Omega_{b}, h\right)=(1.0,0.76,0.24,0.041,0.73)$, and assumed a relative abundance of deuterium $n[\mathrm{D}] / n=2.78 \cdot 10^{-5}[16]$.

\section{Model of a protogalaxy and numerical methods}

This section presents a brief description of the main model parameters and numerical methods applied (more detail may be found in [17, 19]), as well as the initial conditions used.

\subsection{Main Parameters}

In the model considered, the protogalaxy consists of gas surrounded by a spherically symmetric halo of dark matter. The dark-matter density profile corresponds to a modified isothermal sphere:

$$
\rho_{h}(r)=\frac{\rho_{0}}{1+\left(r / r_{0}\right)^{2}},
$$

where $r_{0}$ and $\rho_{0}$ are the radius of the nucleus and the central density, respectively. We assumed a total mass of the protogalaxy (dark halo and gas) of $M_{\mathrm{h}}=10^{7} M_{\odot}$; for redshift $z=12$, this corresponds to $3 \sigma$ perturbations in a $\Lambda$ CDM model with the parameters derived from the three-year observations of the CMB radiation by the WMAP satellite [16]. Such a protogalaxy has a virial radius $r_{\mathrm{v}}=520 \mathrm{pc}$ (for virial relations, see, for instance, [20]). Simple estimates $[21,22]$ indicate that $10^{7} M_{\odot}$ protogalaxies at redshift $z=12$ cooled efficiently and produced the conditions necessary for the birth of first-generation stars. Because of the low efficiency of the fragmentation of the primary gas, we assume that in the low-mass protogalaxies initially appears only one star (see, e.g., [20]), although under certain conditions, in particular in rotating protogalaxies, there may appear a group of stars [19].

Since the minimum temperature of the primordial gas at large redshifts varies from 40 to 200 $\mathrm{K}$ (it is determined by the efficiency of the formation of $\mathrm{H}_{2}$ and HD molecules [21]), the accretion rates onto protostellar cores were higher than for the modern elemental abundances $\left(\dot{M} \sim c_{s}^{3}\right)$. Therefore, first generation stars were much more massive than stars of subsequent generations. Numerical models suggest that their masses varied over a wide range of $\sim 10-10^{3} M_{\odot}$ (see, for instance, [20]). The massive stars formed from the primordial gas were much brighter than stars of subsequent generations containing heavy elements, since the $p-p$ chain requires higher temperatures than the $\mathrm{CNO}$ cycle. Of the total mass range, we shall study the ionization zones 
for 25, 40, 120, and $200 M_{\odot}$ stars. We made this choice because these stars have appreciably different characteristics (see the Table), and such stars ultimately explode as supernovae, but do not collapse into black holes, as is the case with the stars $M>260 M_{\odot}$ [23].

Table 1: Main characteristics of the first stars [24].

\begin{tabular}{ccc}
\hline \hline Mass, $M_{\odot}$ & Lifetime, Myr & $\dot{N}_{H}, \mathrm{~s}^{-1}$ \\
\hline 25 & 6.459 & $7.583 \times 10^{48}$ \\
40 & 3.864 & $2.469 \times 10^{49}$ \\
120 & 2.521 & $1.391 \times 10^{50}$ \\
200 & 2.204 & $2.624 \times 10^{50}$ \\
\hline \hline
\end{tabular}

\subsection{Numerical Methods}

The gas dynamics in the protogalaxy model considered is described using the usual gas-dynamical equations in cylindrical coordinates in the approximation of axial symmetry $(z, r, \phi)$, which were solved numerically using a finite-difference method with an operator splitting technique [25]. A piecewise-parabolic, third-order interpolation scheme was used for the gas transport [26].

To obtain the initial distribution of the gas density in the external gravitational potential of the dark matter $\Phi_{h}$, the equilibrium equations in cylindrical coordinates $(z, r)$ were solved numerically. The gas was assumed to be neutral, to have molecular mass $\mu=1.22$, and to be isothermal, with the temperature equal to the virial value $T_{\text {vir }}(M)$. To solve the equilibrium equations, it is necessary to know the initial velocity of the gas $u_{\phi 0}$, which we obtain from the relation $u_{\phi 0}=\alpha u_{\text {circ }}$, where $u_{\text {circ }}$ is so-called circular velocity of the gas, which is determined by the gravitational potential of the dark matter only (see the virial relation, for example, in [20]), and $\alpha$ is a parameter smaller than unity. The method used to solve the equilibrium equations is described in [27]. The iterations were continued until a gas-density profile corresponding to the mass of gas inside the virial radius was obtained, $M_{\mathrm{g}}=\left(\Omega_{b} / \Omega_{m}\right) M_{\mathrm{h}}$. We restricted our consideration to spheroidal (non-rotating) protogalaxies.

\subsection{Chemical Kinetics and Radiative Transfer}

It was assumed in our model that all chemical agents (atoms, ions, molecules) are passive, i.e., they have a velocity field similar to that of the gas. This makes it possible to consider the gas motion only. The chemical kinetics of the primordial gas takes into account the following main components: $\mathrm{H}, \mathrm{H}^{+}, \mathrm{H}^{-}, \mathrm{He}, \mathrm{He}^{+}, \mathrm{He}^{++}, \mathrm{H}_{2}, \mathrm{H}_{2}^{+}, \mathrm{D}, \mathrm{D}^{+}$and $\mathrm{HD}$. We computed the electron number density by assuming charge conservation. The helium abundance (by mass) was set to $Y_{\mathrm{He}}=0.24$. The rates of chemical reactions for collisional and radiative processes were taken from [28]. The chemical-kinetics equations were solved using the backward differencing scheme [29].

We computed the radiative transfer of the ionizing photons using a grid of radial rays with their origin at the center of the protogalaxy. The number of rays was chosen such that each cell of the two-dimensional grid was crossed by at least 10 rays. In contrast to the threedimensional case, it was not necessary to split the rays as the distance to the central source grows for the homogeneous two-dimensional grid. The ionizing luminosity was isotropically distributed over all rays at the center of the protogalaxy. In each cell, we summed the number of absorbed photons along each radial ray that crosses the cell, then used this number of photons to compute the rates of photochemical reactions and the corresponding heating rate of the gas. The radiative transfer was computed for three spectral ranges: from the threshold for hydrogen 
ionization to the threshold for single ionization of helium (13.6-24.6 eV), from the threshold for single ionization of helium to the threshold for double ionization of helium (24.6-54.4 eV), and from the threshold for double ionization of helium to infinity. The spectral integration assumed that the spectrum of the central star did not change during the transfer process within each of the three spectral ranges, though the relative weights of these ranges changes during the computations.

The method we used was tested many times, both via comparison with the exact results of simple tests for the velocity of propagation of the ionization front in the spherically symmetric case, and via comparison with other codes for cosmology applications [18].

\subsection{Initial Conditions}

To improve the resolution at the center of the protogalaxy, we applied a nonuniform computational grid [19]. The grid spacing can be controled using a coefficient A: reducing A increases the resolution in inner regions of the computational grid and reduces the resolution in outer regions. We used $A=1.5$ and a $900 \times 900$ grid, which provides a physical resolution better than $0.1 \mathrm{pc}$ in the inner $10 \mathrm{pc}$, which decreases to $\sim 1 \mathrm{pc}$ at $r \simeq 200 \mathrm{pc}$. The nonuniform spacing is similar along both $z$ and $r$.

Our computations assumed that the dark matter in the protogalaxy at redshift $z=12$ was already virialized, and that its configuration can already be described by (1). Later, the gas in the protogalaxy cools and the concentration of gas in the central region grows, becoming $\sim 10^{8} \mathrm{~cm}^{-3}$ in our computations. Since physical processes that we did not take into account in our model become important at higher gas concentrations, we assumed that a star is born at the center of the protogalaxy when above number density is attained

\section{Numerical computations}

Let us consider the evolution of the ionization zones around first stars with masses of 25 , 40, 120 and $200 M_{\odot}$ in spheroidal (non-rotating) protogalaxies with total masses of $10^{7} M_{\odot}$ (including dark and barionic matter) that are virialized by redshift $z=12$. Figure 1 shows the distributions of the gas density and temperature around 25, 40, 120 and $200 M_{\odot}$ stars in the central region of a protogalaxy at $t=0.99 t_{l f}^{*}$, where $t_{l f}^{*}$ is the main-sequence lifetime of the star (Table 1, [24]). It is clear that the distribution of the gas in the vicinities of the massive stars depends strongly on the mass of the star. A $200 M_{\odot}$ star ionizes and heats the gas not only in the region $r<0.1 \mathrm{kpc}$, but also outside the virial radius for a $10^{7} M_{\odot}$ protogalaxy, as can be seen in the upper part of Fig. 2. Small fluctuations are visible in the distributions of the density and temperature inside the ionization zone of such a star, and the average temperature of the gas is $\sim 3 \times 10^{4} \mathrm{~K}$. The ionization zone for a lower mass star $\left(120 M_{\odot}\right)$ is much smaller, $r_{\mathrm{HII}} \sim 0.1 \mathrm{kpc}$; the fluctuations of the gas density and temperature are somewhat higher than in the gas around a $200 M_{\odot}$ star, but remain small. "Lobes" of hot (rarefied) and cool (dense) gas are visible in the ionization region, which arise due to fragmentation of the envelope formed by the shock and subsequent compression of these fragments by radiation. The formation of the "lobes" is related to the shadow of the envelope fragments [3].

The ionization zones for 25 and $40 M_{\odot}$ stars are much smaller than the virial radius of the protogalaxy: $\sim 30$ and $40 \mathrm{pc}$, respectively. At the same time, a D-front probably forms around such stars, in contrast to the weak R-fronts that form around more massive stars (120 and $\left.200 M_{\odot}\right)$. Figures 1 and 2 clearly show the ionization front itself and a distinct shock propagating with supersonic velocity relative to the gas around the 25 and $40 M_{\odot}$ stars, and a propagating ionization shock around the 120 and $200 M_{\odot}$ stars. The gas density behind the fronts is fairly high, and cooling effects immediately begin to become important, as is manifest by the enhanced 

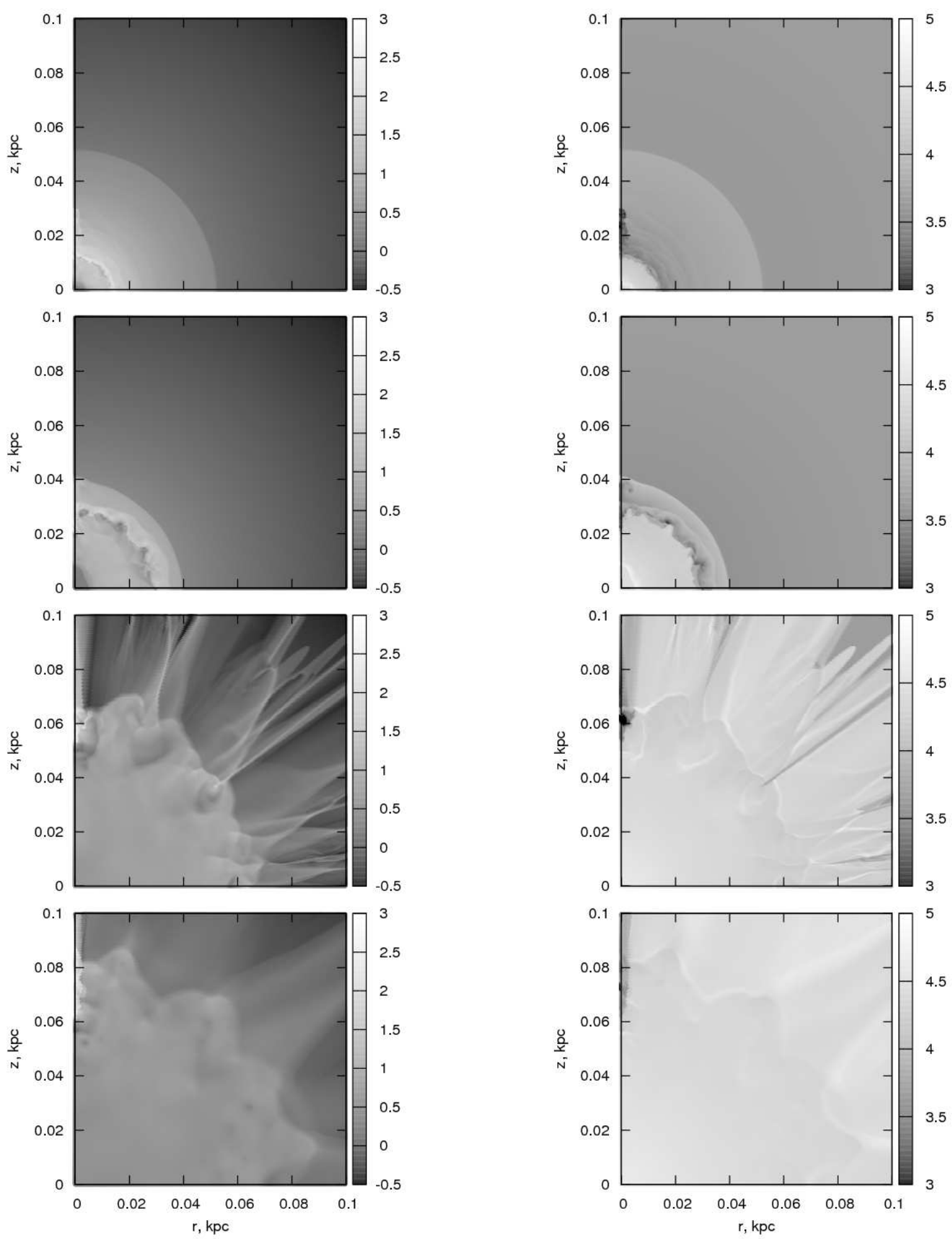

Figure 1: Maps of the distributions of the gas density (left) and temperature (right) in relative units around $25,40,120$, and $200 M_{\odot}$ stars (top to bottom) at time $t=0.99 t_{l f}^{*}$, where $t_{l f}^{*}$ is stellar lifetime on the main sequence (table) [24], in the central region of a spheroidal protogalaxy with total mass $M=10^{7} M_{\odot}$ ( spin momentum $\lambda=0$ ). 

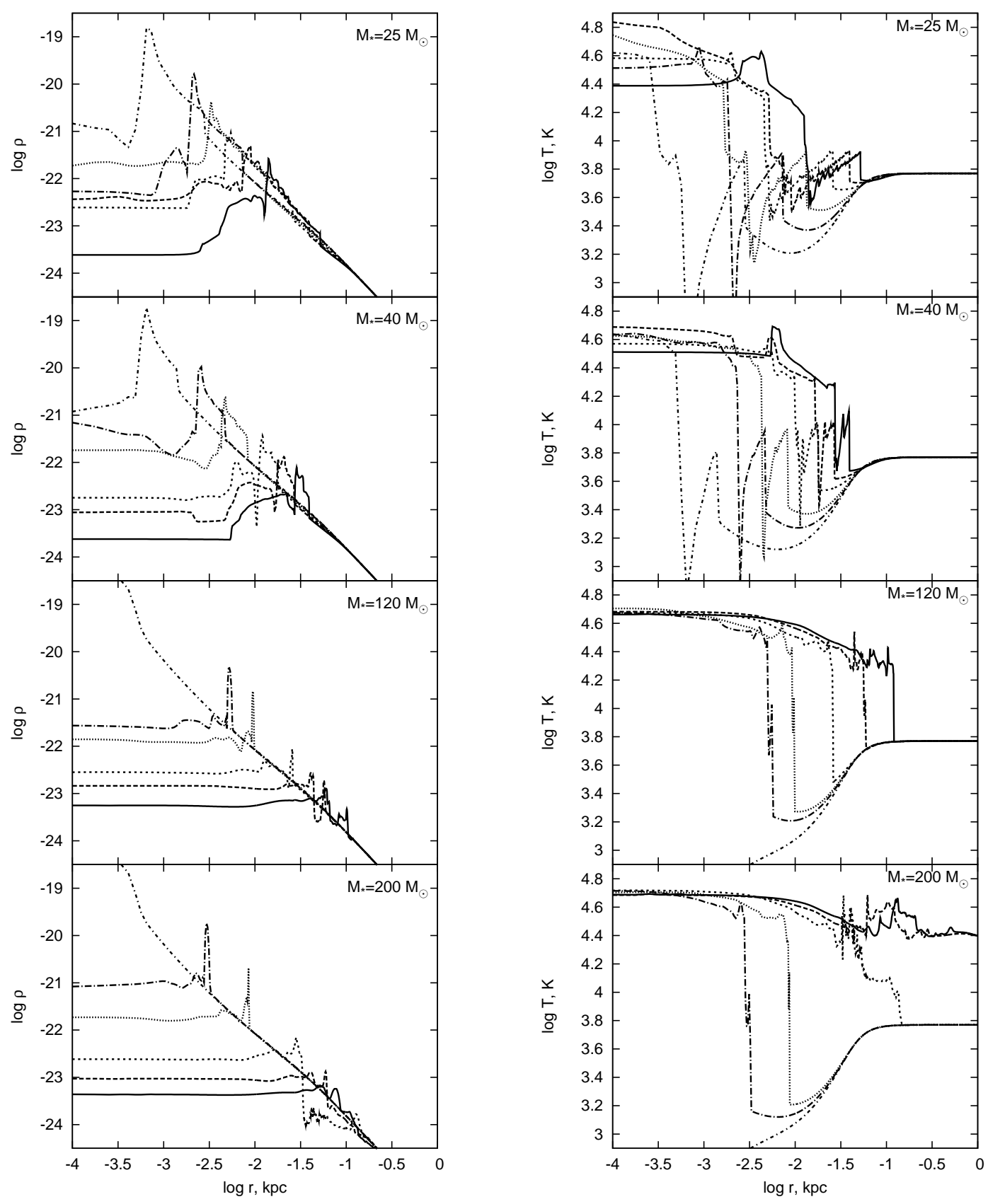

Figure 2: Radial distributions of the gas density (left) and temperature (right) around $M_{*}=$ 25, 40, 120, and $200 M_{\odot}$ stars (top to bottom) along a ray from the center of a protogalaxy with total mass $M=10^{7} M_{\odot}$ at an angle $\pi / 4$ to the vertical axis at times $0.1,0.2,0.3,0.55$, 0.8, $0.99 t_{l f}^{*}$ (top to bottom). 
density of the gas envelope formed by the ionization front. Figure 2 shows radial profiles of the gas density and temperature along a ray directed from the center of the protogalaxy at an angle of $\pi / 4$ relative to the vertical axis at times $t=0.1,0.2,0.3,0.55,0.8,0.99 t_{l f}^{*}$. For the ionization zones around the 120 and $200 M_{\odot}$ stars, the density jump in the envelope created by the R-front exceeds an order of magnitude at $t \lesssim 0.2 t_{l f}^{*}$; later, the envelope smears out (it is disrupted). For the zones around 25 and $40 M_{\odot}$ stars, the profiles in Fig. 2 show that the strong D-front formed before $0.1 t_{l f}^{*}\left(\sim 0.4\right.$ and $0.65 \mathrm{Myr}$, respectively, for 25 and $40 M_{\odot}$ stars $)$ and the distribution of gas behind the front does not show any inhomogeneities. However, at a time of $\sim 0.3 t_{l f}^{*}$, density and temperature fluctuations arise behind the front. These fluctuations may be related to two processes with different natures: thermal instability and Rayleigh-Taylor instability.

The growth of instabilities in the ionization zones depends on the mass of the central star. This is obviously due to the different numbers of ionizing photons emitted by the stars. A $200 M_{\odot}$ star emits 40 times more photons than a $25 M_{\odot}$ star, and the numbers of photons emitted pairs 25 and $40 M_{\odot}$ and 120 and $200 M_{\odot}$ stars differ by factors of two to three (see the table). Therefore, the nature of the instabilities in these pairs is expected to be similar, as is seen in Figs. 1 and 2. On one hand, low-mass stars emit fewer ionizing photons, resulting in a lower velocity for the ionization-front expansion than in the case of massive stars. Thus, if the zone expands into a region with a power-law density profile, at similar ages, the gas density at the front of the ionization zone will be higher for low-mass stars, making the timescales for cooling and the growth of Rayleigh-Taylor instability shorter. On the other hand, the lifetimes of lowmass stars are longer, making the time available for the growth of instabilities longer. Therefore, more developed instability is expected in the vicinities of lower mass stars $\left(25-40 M_{\odot}\right)$, as is clearly visible in Figs. 1 and 2.

To study variations of the physical parameters along the ionization front, let us find the radius $r_{m}$ corresponding to the maximum density along a ray directed from the center of the protogalaxy at an angle $\pi / 4$ to the vertical axis. We assume that the circle with radius $r_{m}$ corresponds to the region of the ionization front, which, in general, is close to the real situation (Fig. 2). Next, we identify the cells of the computational grid that are located closest to the radius $r_{m}$; the polar angle $\phi$ is measured from the vertical axis. As a result, we obtain the axial distribution of the gas along a circle with radius $r_{m}$.

Figure 3 presents the distributions of the density (lower) and temperature (upper) along circles with radii $r_{m}=28.6 \mathrm{pc}, r_{m}+\delta r$ and $r_{m}-\delta r$, where $2 \delta r=r_{m} / 12$, for a $40 M_{\odot}$ star at $t=0.99 t_{l f}^{*}$. The short-wavelength perturbations are modulated by longer-wavelength ones. Perturbations with different wavelengths probably arise due to the Rayleigh-Taylor instability, which has an increment $\gamma_{R T} \sim \sqrt{k g}$, where $k$ is the wave vector, $g \sim \chi^{-1} v_{r}^{2} / R$ is the acceleration of the envelope, and $\chi$ is the ratio of the densities behind and ahead of the front. For the radius $r_{m} \simeq R=30 \mathrm{pc}$, a gas velocity $v \sim 10 \mathrm{~km} \mathrm{~s}^{-1}$ and $\chi \sim 10$ the maximum wavelength for which $\gamma_{R T} t \sim 1$ at $t \simeq 4 \mathrm{Myr}$ is $\sim 15 \mathrm{pc}$; this value is close to the scale of the largest perturbations, $r_{m} \phi \sim 12-15 \mathrm{pc}$, shown in Fig. 2. Short-wavelength perturbations arose earlier, when the radius of the ionized envelope was smaller. The appearance of the smallest perturbations, with $\lambda \lesssim 1 \mathrm{pc}$, is probably related to thermal instability, since the ionization zone is non-stationary and radiative heating is not completely balanced by cooling. The cooling timescale appears to be fairly short, $t_{c} \sim 10^{11} \mathrm{~s}$, and the size of the perturbation is close to $\lambda_{t} \sim c_{s} t_{c} \sim 0.03 \mathrm{pc}$, comparable to the size of the computational grid cells. Moreover, Rayleigh-Taylor instability can also be significant on small scales, since its increment grows as $\lambda^{-1 / 2}$.

Let now describe the global characteristics of the ionization zones. Figure 4 shows the evolution of the mass fraction comprised by the gas with relative electron number density exceeding 0.1 , in the region inside radii of $r_{v i r}$ (solid) and $0.1 r_{v i r}$ (dashed); the mass fraction of gas having a relative electron number density of $10^{-3}$ in these same regions (dotted and dash-dotted, respectively); and the mass fraction of gas with temperatures higher than $2 \times 10^{4} \mathrm{~K}$ (dash- 


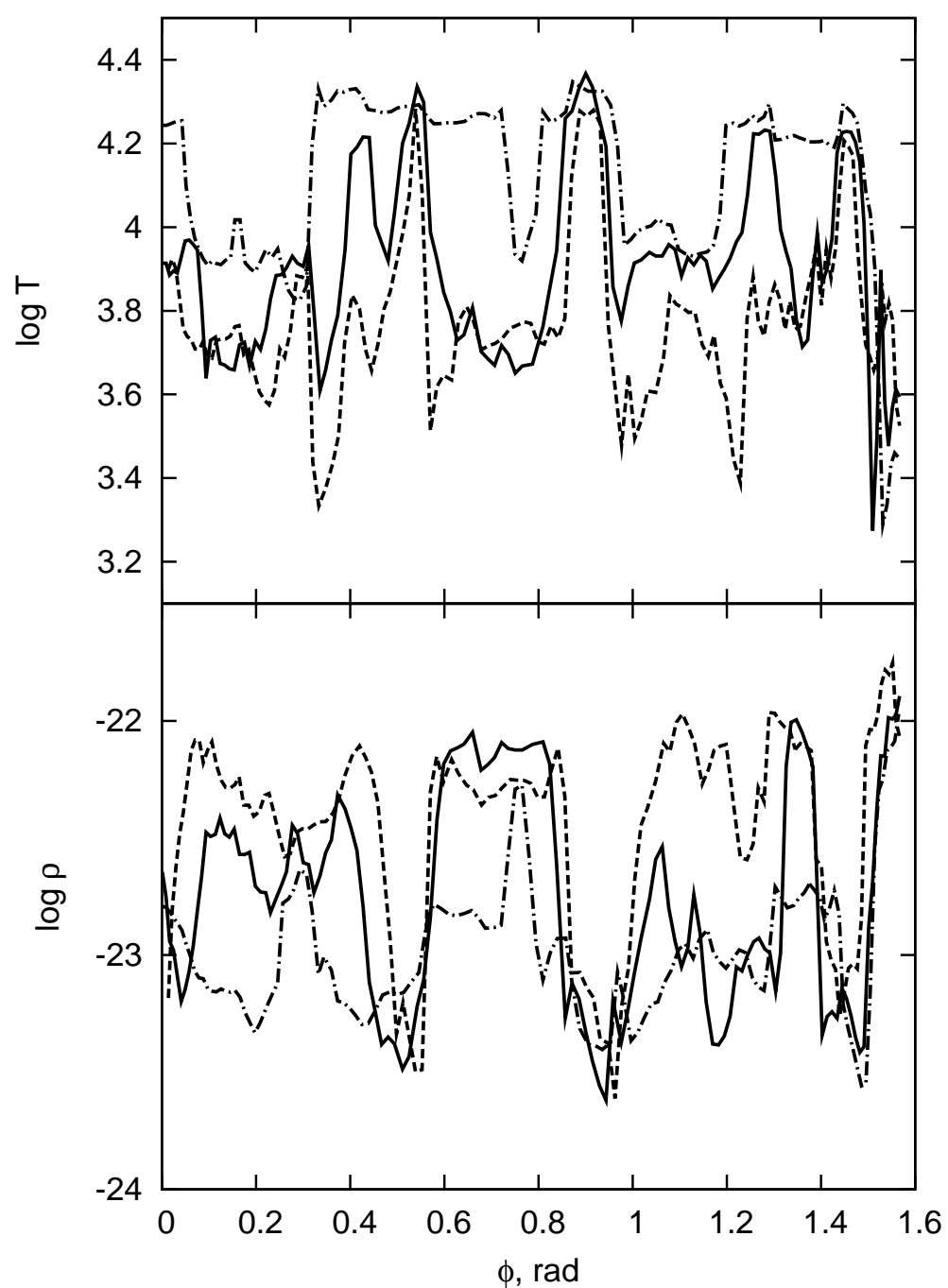

Figure 3: Distributions of the density (lower) and temperature (upper) along circles with radii of $r_{m}=28.6 \mathrm{pc}$ (solid), $r_{m}+\delta r$ (dashed), and $r_{m}-\delta r$ (dash.dotted), where $2 \delta r=r_{m} / 12$, for a $40 M_{\odot}$ star at $t=0.99 t_{l f}^{*}$. 


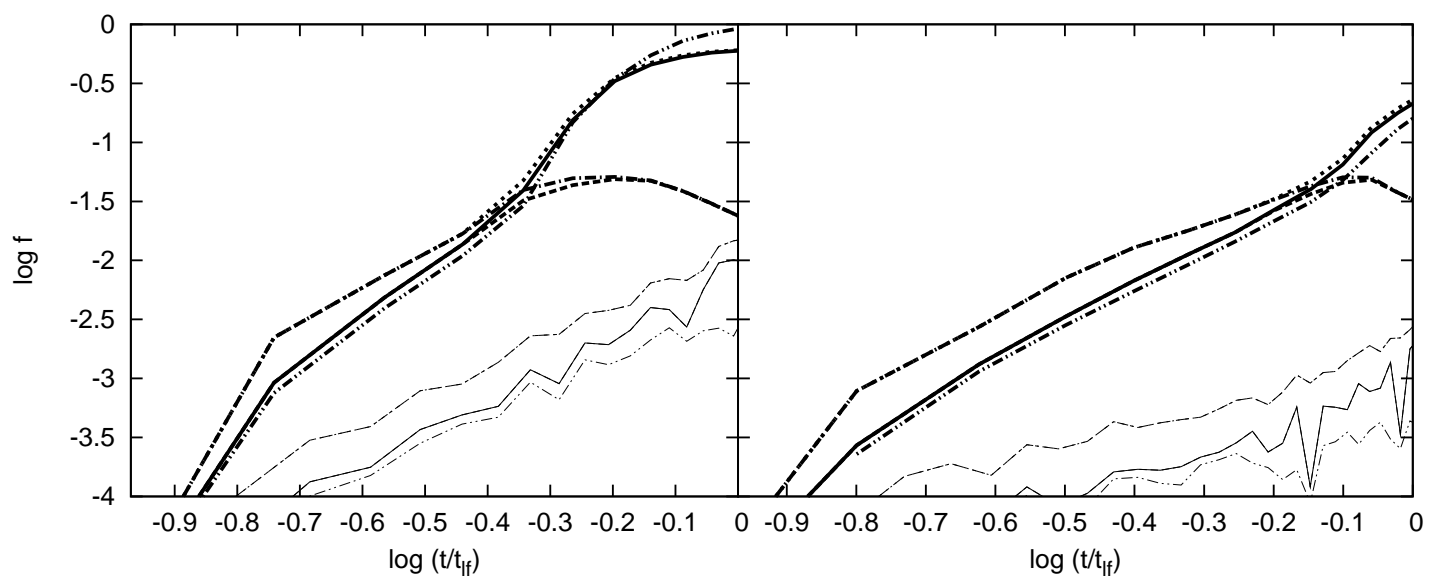

Figure 4: Evolution of the mass fraction of the gas having a relative electron number density exceeding 0.1 in the regions inside radii of $r_{v i r}$ (solid) and $0.1 r_{v i r}$ (dashed); mass fraction of gas having a relative electron number density of $10^{-3}$ in these same regions (dotted and dashdotted, respectively); mass fraction of gas with temperatures higher than $2 \times 10^{4} \mathrm{~K}$ (dash-double dotted). The left panel shows these dependences for $200 M_{\odot}$ (thick solid) and $40 M_{\odot}$ (thin) stars. The right panel shows the same for $120 M_{\odot}$ (thick solid) and $25 M_{\odot}$ (thin) stars. The curves for radii of $r_{v i r}$ and $0.1 r_{v i r}$ merge for the 25 and $40 M_{\odot}$ stars.

double dotted). For the 120 and $200 M_{\odot}$ stars (thick solid), the mass of ionized gas increases with time, and exceeds $10 \%$ of the mass of the protogalaxy at times $t=0.8 t_{l f}^{*}$ and $0.5 t_{l f}^{*}$, respectively, while the radius of the ionization zone exceeds $50 \mathrm{pc}\left(\sim 0.1 r_{v i r}\right)$. At these times, the degree of ionization inside the virial radius is either less than 0.001 or greater then 0.1 (the curves are very close), whereas the temperature of the ionized gas exceeds $2 \times 10^{4} \mathrm{~K}$. The gas in a protogalaxy with a $200 M_{\odot}$ star is appreciably ionized at $0.8 t_{l f}^{*}$, while only one-third of the gas is ionized in a protogalaxy with a $120 M_{\odot}$ central star at the end of the star's lifetime. This provides evidence that ionizing photons from stars with $M_{*} \lesssim 120 M_{\odot}$ cannot leave $M \gtrsim 10^{7} M_{\odot}$ protogalaxies. If the mass of the central stars is 25 and $40 M_{\odot}$ (thin curves), the gas in protogalaxies with $M \gtrsim 10^{7} M_{\odot}$ remains essentially neutral, and the ionization zone does not extend behind $0.1 r_{v i r}$. There exists a significant fraction of the gas mass with degrees of ionization $0.001<x_{e}<0.1$. It is clear that this gas could be associated with gas that is behind the shock, but has passed through the ionization front. This conclusion is supported by the fact that the mass of gas with $T>2 \times 10^{4} \mathrm{~K}$ is approximately equal to the mass of gas with $x_{e}>0.1$ (the solid and dash-double dot curves are very close).

To conclude, we note that the efficiency of the ejection of heavy elements by supernovae is probably much higher in protogalaxies with $M \gtrsim 10^{7} M_{\odot}$ containing stars with $M_{*} \sim 120-$ $200 M_{\odot}$ than in such protogalaxies containing stars with $M_{*} \lesssim 40 M_{\odot}$, since, first, the ionization fronts from more massive stars substantially reduce the average density of the gas in the protogalaxy and, second, the energy released by the explosions of massive stars is a factor of $\sim 3-$ 10 higher than the corresponding energy for less massive stars [23]. Accordingly, the radiative phase of a supernova remnant will start earlier in protogalaxies with lower-mass stars, and the development of Rayleigh-Taylor instability in the envelope is likely, so that a significant fraction of the heavy elements will be locked up in the protogalaxy as a consequence. 


\section{Conclusions}

We have considered the dynamical, thermal, and chemical evolution of gas associated with the formation of the ionization zones around first stars with masses of $M_{*} \sim 25-200 M_{\odot}$ in protogalaxies with masses of $M \sim 10^{7} M_{\odot}$ at redshift $z=12$, and investigated the conditions for the development of instabilities in the ionization zones. We have shown the following.

1. Rayleigh-Taylor and thermal instabilities develop in the ionization zones, which are especially strong around 25-40 $M_{\odot}$ stars and less important for stars with masses $\sim 120 M_{\odot}$; if the stars are more massive $\left(\sim 200 M_{\odot}\right)$, the flux of ionizing photons over the entire lifetime of a star turns out to be sufficient to create a weak ionization R-front, behind which the instabilities do not have time to grow.

2. The gas in a protogalaxy with $M \sim 10^{7} M_{\odot}$ with a $200 M_{\odot}$ star is completely ionized by the end of the star's lifetime, while only one-third of the gas is ionized in the case of a $120 M_{\odot}$ star. Thus, ionizing photons from stars with $M_{*} \lesssim 120 M_{\odot}$ are not able to leave protogalaxies with $M \gtrsim 10^{7} M_{\odot}$. If the mass of the central star is 25 or $40 M_{\odot}$, the gas in a protogalaxy with this mass remains essentially neutral.

3. After the supernova explosion, heavy elements will be efficiently ejected from protogalaxies with $M \sim 10^{7} M_{\odot}$ containing stars with masses $\sim 120-200 M_{\odot}$. The ejection efficiency is negligible for stars of lower massses $\left(25-40 M_{\odot}\right)$; i.e., after the explosions of 25-40 $M_{\odot}$ stars, a significant fraction of heavy elements will be locked inside the protogalaxy.

\section{Acknowledgements}

This work was supported by the Russian Foundation for Basic Research (project code 09-0200933), the Austrian Scientific Foundation (FWF, project code M 1255-N16), the Ministry of Education and Science of the Russian Federation (the Departmental Analytical Targeted Program The Development of the Potential of Higher Education (projects RNP-2.1.1.5940, RNP 2.1.1/11879, state contract P-685). EOV acknowledges support from the Russian Foundation for Basic Research, project codes 11-02-90701, 11-02-01332) and the Dynasty foundation. Yu.A.S. acknowledges the Russian Foundation for Basic Research (project code 11-02-97124). Computations were performed using computer clusters of the Computing Center of the Southern Federal University and the Center for Multiaccess to Computational Resources of the Southern Federal University. E.O.V. thanks V.N. Datsyuk for useful discussions and advise.

\section{References}

[1] E.T. Vishniac, Astrophys. J. 274, 152 (1983)

[2] M.-M. Mac Low and M.L. Norman, Astrophys. J. 407, 207 (1993)

[3] R.J.R. Williams, Mon. Not. Roy. Astron. Soc. 310, 789 (1999)

[4] M.A. de Avillez, M.-M. Mac Low, Astrophys. J. 581, 1047 (2002)

[5] J. Scalo, and B.G. Elmegreen, Ann. Rev. Astron. Astrophys. 42, 275 (2004)

[6] D. Whalen, T. Abel, M.L. Norman, Astrophys. J. 610, 14 (2004)

[7] M.A. Alvarez, V. Bromm, P.R. Shapiro, Astrophys. J. 639, 621 (2006) 
[8] B. W. O’Shea, T. Abel, D. Whalen, M.L. Norman, Astrophys. J. Lett. 628, 5 (2005)

[9] T. Abel, J.H. Wise, G.L. Bryan, Astrophys. J. Lett. 659, 87 (2007).

[10] T. Kitayama, N. Yoshida, H. Susa, M. Umemura, Astrophys. J. 613, 631 (2004)

[11] H. Yajima, M. Umemura, M. Mori, T. Nakamoto, Mon. Not. Roy. Astron. Soc. 398, 715 (2009)

[12] J.H. Wise, and R. Cen, Astrophys. J. 693, 984 (2009)

[13] T.H. Greif, J.L. Johnson, R.S. Klessen, V. Bromm, Mon. Not. Roy. Astron. Soc. 399, 639 (2009)

[14] A. Mesinger, G.L. Bryan, Z. Haiman, Mon. Not. Roy. Astron. Soc. 399, 1650 (2009)

[15] D. Whalen, and M.L. Norman, Astrophys. J. 673, 664 (2008)

[16] D.N. Spergel, R. Bean, O. Doré, et. al., Astrophys. J. Suppl. Ser. 170, 377 (2007)

[17] E.O. Vasiliev, E.I. Vorobyov, Yu.A. Shchekinov, Astron. and Astrophys. 489, 505 (2008)

[18] I.T. Iliev, B. Ciardi, M.A. Alvarez, A. Maselli, A. Ferrara et al. , Mon. Not. Roy. Astron. Soc. 371, 1057 (2006)

[19] E.O. Vasiliev, E.I. Vorobyov, Yu.A. Shchekinov, Astron. Reps 54, 890 (2010)

[20] B. Ciardi and A. Ferrara, Space Sci. Rev. 116, 625 (2005)

[21] Yu.A. Shchekinov and E.O. Vasiliev, Mon. Not. Roy. Astron. Soc. 368, 454 (2006)

[22] M. Tegmark, J. Silk, M.J. Rees, A. Blanchard, T. Abel, F. Palla, Astrophys. J. 474, 1 (1997)

[23] A. Heger and S. Woosley, Astrophys. J. 567, 532 (2002)

[24] D. Schaerer, Astron. and Astrophys 382, 28 (2002)

[25] M.L. Norman, J.R. Wilson, R. Barton, Astrophys. J. 239, 968 (1980)

[26] P. Collela and P.R. Woodward J. Comp. Phys. 54, 174 (1984)

[27] E.I. Vorobyov, U. Klein, Yu.A. Shchekinov, J. Ott, Astron. and Astrophys. 413, 939 (2004)

[28] T. Abel, P. Anninos, Yu. Zhang, M.L. Norman, New Astron. 2, 181 (1997)

[29] E. S. Oran and J. P. Boris, Numerical Simulation of Reactive Flow (Elsevier, New York, 1987; Mir, Moscow, 1990). 